\title{
Defective choosability of graphs without small minors
}

\author{
Rupert G. Wood and Douglas R. Woodall \\ School of Mathematical Sciences, \\ University of Nottingham, \\ Nottingham NG7 2RD, UK \\ rupert.wood@gmail.com, douglas.woodall@nottingham.ac.uk
}

Submitted: Jan 9, 2008; Accepted: Jul 22, 2009; Published: Jul 31, 2009

Mathematics Subject Classification: 05C15

\begin{abstract}
For each proper subgraph $H$ of $K_{5}$, we determine all pairs $(k, d)$ such that every $H$-minor-free graph is $(k, d)^{*}$-choosable or $(k, d)^{-}$-choosable. The main structural lemma is that the only 3 -connected $\left(K_{5}-e\right)$-minor-free graphs are wheels, the triangular prism, and $K_{3,3}$; this is used to prove that every $\left(K_{5}-e\right)$-minor-free graph is 4 -choosable and $(3,1)$-choosable.
\end{abstract}

Keywords: List colouring; Defective choosability; Minor-free graph

\section{Introduction}

Throughout this paper, all graphs are simple. A subgraph of a vertex-coloured graph is monochromatic if all its vertices have the same colour. A (possibly improper) vertex $k$-colouring of a graph $G$ is a $(k, d)^{*}$-colouring if no vertex has more than $d$ neighbours with the same colour as itself, i.e., there is no monochromatic subgraph isomorphic to $K_{1, d+1}$; and it is a $(k, d)^{-}$-colouring if there is no monochromatic path $P_{d+2}$ with $d+1$ edges and $d+2$ vertices. The superscripts * and ${ }^{-}$are to remind us that the forbidden monochromatic subgraphs are stars and paths, respectively. However, we may omit the superscript if $d \leqslant 1$, since $(k, 0)^{*}$-colourings and $(k, 0)^{-}$-colourings are both the same as (proper) $k$-colourings, and $(k, 1)^{*}$-colourings are also the same as $(k, 1)^{-}$-colourings.

A list-assignment $L$ to (the vertices of) $G$ is an assignment of a 'list' (set) $L(v)$ of colours to every vertex $v$ of $G$; and a $k$-list-assignment is a list-assignment such that $|L(v)| \geqslant k$ for every vertex $v$. If $L$ is a list-assignment to $G$, then an $L$-colouring of $G$ is a colouring (not necessarily proper) in which each vertex receives a colour from its own list. An $(L, d)^{*}$-colouring or $(L, d)^{-}$-colouring is an $L$-colouring in which there is no monochromatic star $K_{1, d+1}$ or path $P_{d+2}$, respectively. A graph $G$ is $(k, d)^{*}$-choosable or $(k, d)^{-}$-choosable if it has an $(L, d)^{*}$-colouring or an $(L, d)^{-}$-colouring, respectively, 
whenever $L$ is a $k$-list-assignment to $G$. Then $(k, 0)^{*}$-choosable and $(k, 0)^{-}$-choosable both mean the same as $k$-choosable, and $(k, 1)^{*}$-choosable means the same as $(k, 1)^{-}$-choosable and may be called simply $(k, 1)$-choosable.

We write $(a, b) \geqslant(c, d)$ if $a \geqslant c$ and $b \geqslant d$. It is easy to see that if $\left(k^{\prime}, d^{\prime}\right) \geqslant(k, d)$ and a graph $G$ is $(k, d)^{*}$-choosable or $(k, d)^{-}$-choosable, then $G$ is $\left(k^{\prime}, d^{\prime}\right)^{*}$-choosable or $\left(k^{\prime}, d^{\prime}\right)^{-}$-choosable, respectively. Thus to specify all pairs $(k, d)$ for which a graph has one of these properties, it suffices to specify all the minimal such pairs. In [11], the second author determined and tabulated, for every graph $H$ with at most five vertices, all the pairs $(k, d)$ such that every $H$-minor-free graph is $(k, d)^{*}$-colourable or $(k, d)^{-}$-colourable. The purpose of the present paper is to do the same for $(k, d)^{*}$-choosability and $(k, d)^{-}$choosability, and this purpose is achieved except that we have not been able to determine whether all $K_{5}$-minor-free graphs are $(4,1)$-choosable, or even whether there is any $d$ for which they are all $(4, d)^{-}$-choosable. Our results can be summarized as follows.

Theorem 1.1. (Summary Theorem.) Let $H(i)(1 \leqslant i \leqslant 30)$ be any one of the 30 connected graphs with between 2 and 5 vertices, as listed in column 1 of Table 1 . Then the statements 'Every $H(i)$-minor-free graph is $(k, d)^{*}$-choosable' and 'Every $H(i)$-minor-free graph is $(k, d)^{-}$-choosable' are true if and only if $(k, d)$ is greater than or equal to one of the values listed in the appropriate row and column of Table 1.

If Table 1 is compared with the analogous table in [11], it will be seen that there are two main differences. Firstly, $H(17)$-minor-free graphs and $H(18)$-minor-free graphs are all $(2,1)$-colourable, but they are not all $(2,1)$-choosable, and indeed are not all $(2, d)^{*}$ choosable for any fixed value of the so-called 'defect' $d$. They have thus dropped down one category in Table 1 compared with [11]. (In view of this, the graphs $H(13)-H(18)$ have been renumbered here compared with [11].)

Secondly, as a consequence of the 4-colour theorem, every $K_{5}$-minor-free graph is 4-colourable. However, it is known $[4,5,9]$ that not every planar graph, and hence not every $K_{5}$-minor-free graph, is 4-choosable. Thomassen [8] proved that every planar graph is 5-choosable, and Škrekovski [6] deduced from this that every $K_{5}$-minor-free graph is 5 -choosable. It is not known whether or not every planar graph (or every $K_{5}$-minor-free graph) is $(4,1)$-choosable, but the $(3,2)^{*}$-choosability of planar graphs was proved by Škrekovski [7]; see ([12], section 4) for further information about planar, $K_{5}$-minor-free and $K_{3,3}$-minor-free graphs.

The rest of this paper is devoted to a proof of Theorem 1.1. For each row of Table 1 labelled $H(i)(1 \leqslant i \leqslant 30)$, and for each value $(k, d)$ in column 2 or 3 of that row, it suffices to provide an argument showing that every $H(i)$-minor-free graph is $(k, d)^{*}$-choosable or $(k, d)^{-}$-choosable, respectively, as well as examples to show that this would not follow if $(k, d)$ were replaced by $(k-1, d)$ or $(k, d-1)$. The arguments are indexed in column 4 of the table and explained in sections 3 and 4 . The examples are indexed in column 5 of the table and explained in section 2 .

An argument or example labelled $X_{k, d}^{*}$ proves or disproves, respectively, the assertion that every graph in the given class is $(k, d)^{*}$-choosable. Arguments and examples labelled 


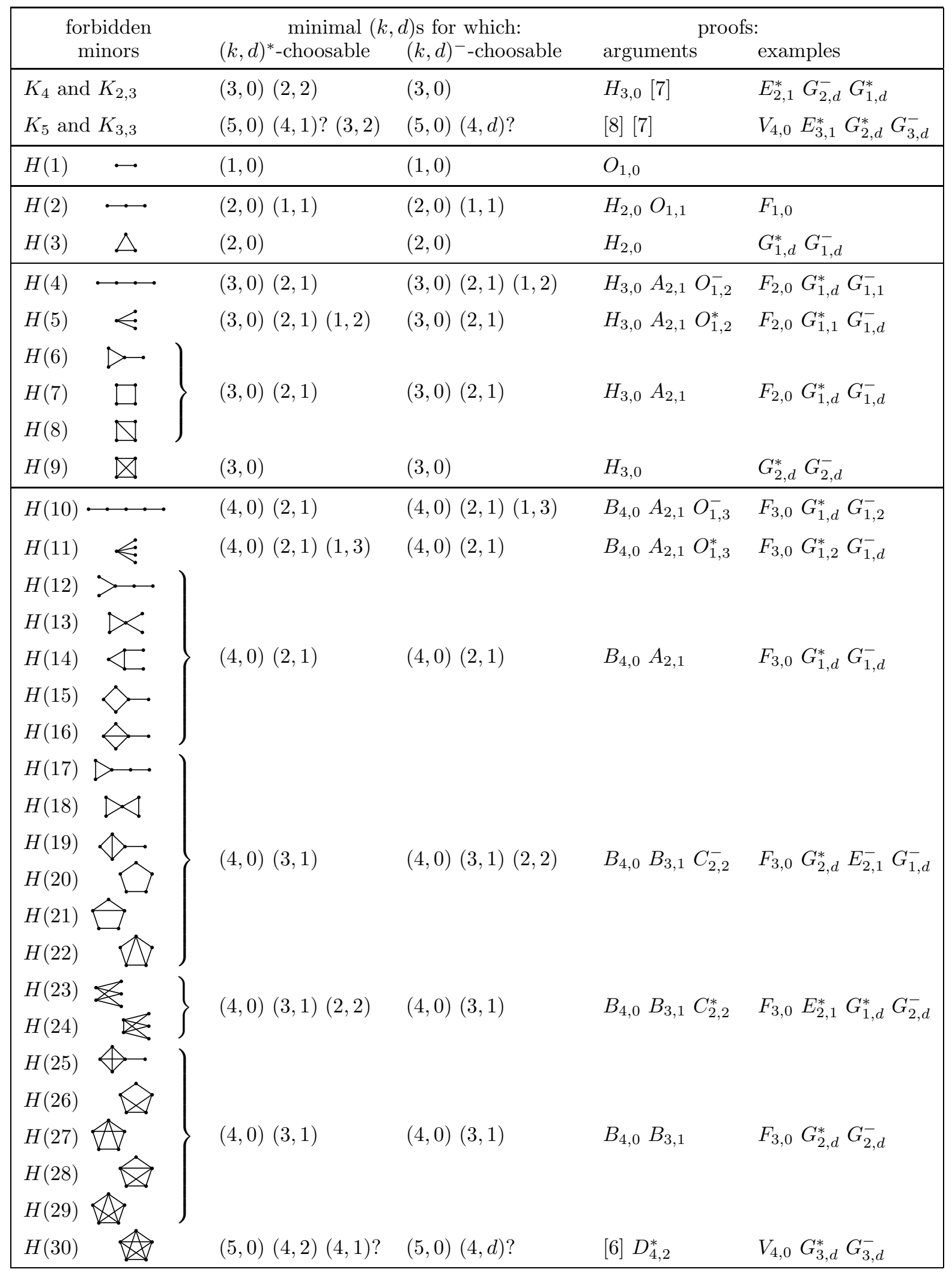

Table 1 
$X_{k, d}^{-}$do the same for $(k, d)^{-}$-choosability, while those labelled $X_{k, d}$ do the same for both $(k, d)^{*}$-choosability and $(k, d)^{-}$-choosability.

\section{Examples}

In this section we present examples to show that the results listed in Table 1 are sharp. Note that if $H(i)$ is a minor of $H(j)$, then every $H(i)$-minor-free graph is also $H(j)$ minor-free, and so an example $X_{k, d}$ that is $H(i)$-minor-free will work for the class of $H(j)$-minor-free graphs as well.

Examples $\boldsymbol{E}_{\boldsymbol{k}, \mathbf{1}}^{*}$ and $\boldsymbol{E}_{\boldsymbol{k}, \mathbf{1}}^{-}$: We use these names when no new examples are required, since $E_{k, 1}^{*}$ is covered by $G_{k, d}^{-}$and $E_{k, 1}^{-}$is covered by $G_{k, d}^{*}$. We could formally define $E_{k, 1}^{*}:=G_{k, 1}^{-}$and $E_{k, 1}^{-}:=G_{k, 1}^{*}$. For example, to satisfy the requirements of Table $1, E_{2,1}^{*}$ must be a graph that does not have $H(23)$ or $H(24)$ as a minor and is not $(2,1)^{*}$-choosable, while $G_{2,1}^{-}$must be a graph that does not have any of $H(23), \ldots, H(29)$ as a minor and is not $(2,1)^{-}$-choosable; so whatever graph we choose for $G_{2,1}^{-}$will do for $E_{2,1}^{*}$ as well.

Alternatively, we can get simpler examples for $E_{k, 1}^{*}$ by defining $E_{2,1}^{*}:=K_{1}+2 K_{1,2}$ and $E_{3,1}^{*}:=K_{1}+2\left(K_{1}+2 K_{1,2}\right)$, where + denotes 'join'. The former is outerplanar, and so does not have $H(23)\left(K_{2,3}\right)$ or $H(24)$ as a minor, and the latter is therefore planar. The former is not $(2,1)^{*}$-colourable, since whichever colour was given to the $K_{1}$, at least one of the two copies of $K_{1,2}$ would have to have all its vertices coloured with the other colour; thus it is not $(2,1)^{*}$-choosable either. By the same reasoning, $K_{1}+2\left(K_{1}+2 K_{1,2}\right)$ is not $(3,1)^{*}$-colourable and so not $(3,1)^{*}$-choosable.

Example $\boldsymbol{F}_{\boldsymbol{k}, \mathbf{0}}$ : This is defined to be $K_{k+1}$, which is not $k$-colourable, and so not $k$-choosable, and has no minor with $k+2$ vertices.

Example $G_{1, d}^{*}$ : This is defined to be $K_{1, d+1}$, which is not $(1, d)^{*}$-colourable, and so not $(1, d)^{*}$-choosable. It cannot have as a minor any graph containing either a circuit or a path with more than 2 edges, which includes $H(i)$ for $i \in\{3,4,6, \ldots, 10,12, \ldots, 24\}$. Also, $G_{1,1}^{*}\left(K_{1,2}\right)$ does not have $H(5)\left(K_{1,3}\right)$ as a minor, and $G_{1,2}^{*}\left(K_{1,3}\right)$ does not have $H(11)\left(K_{1,4}\right)$ as a minor.

Example $\boldsymbol{G}_{\boldsymbol{k}, \boldsymbol{d}}^{*}(\boldsymbol{k} \geqslant 2)$ : Unlike in [11], the graphs that $G_{2, d}^{*}$ must not have as a minor now include $H(17)$ and $H(18)$. This means that we cannot use the same example as was used for a non- $(2, d)^{*}$-colourable graph in [11], namely $K_{1}+(d+1) K_{1, d+1}$, since it has both $H(17)$ and $H(18)$ as minors when $d \geqslant 1$. Indeed all $H(17)$-minor-free graphs and $H(18)$-minor-free graphs are $(2,1)$-colourable, and hence $(2, d)^{*}$-colourable when $d \geqslant 1$. We thus need a new example, which does not have either $H(17)$ or $H(18)$ as a minor, and is therefore $(2, d)^{*}$-colourable, but not $(2, d)^{*}$-choosable.

So define $G_{k, d}^{*}:=K_{k, k^{k}(k d+1)}$, so that $G_{2, d}^{*}=K_{2,8 d+4}$ and $G_{3, d}^{*}=K_{3,81 d+27}$. Let $G:=G_{k, d}^{*}$ and let the partite sets of $G$ be $X$ and $Y$, where $|X|=k$ and $|Y|=k^{k}(k d+1)$. 
To show that $G$ is not $(k, d)^{*}$-choosable, assign disjoint lists of size $k$ to the $k$ vertices in $X$, and for each of the $k^{k}$ transversals of these $k$ lists, assign that transversal as list to $k d+1$ vertices of $Y$. Then in whatever way the vertices of $X$ are coloured from their lists, there will be $k d+1$ vertices of $Y$ that have no colour in their list that has not already been used on $X$; at least $d+1$ of these vertices must use the same colour, giving a monochromatic $K_{1, d+1}$ in $G$.

To show that $G$ is $K_{k+2^{2}}$-minor-free, we show that every minor of $G$ is $(k+1)$-colourable. Consider the (proper) $(k+1)$-colouring of $G$ in which the vertices of $X$ are coloured $1, \ldots, k$, and all vertices of $Y$ are coloured $k+1$. Whenever an edge of $G$ is contracted, give the new vertex the smaller of the colours of the two vertices that were merged into it. Since there is never more than one vertex with each of the colours $1, \ldots, k$, and no new vertex ever gets colour $k+1$, the resulting colouring is proper.

It remains to show that $G_{2, d}^{*}$ has neither $H(17)$ nor $H(20)$ as a minor, since every graph $H(i)(i \in\{17, \ldots, 22,25, \ldots, 29\})$ where $G_{2, d}^{*}$ is used (apart from $K_{4}$, which we have just dealt with) has one of these as a minor. It is clear that $H(20)\left(C_{5}\right)$ is not a minor of $G_{2, d}^{*}$, since the longest circuit in $K_{2,8 d+4}$ has length 4 . To see that $H(17)$ is not a minor either, it suffices to note that every minor of $K_{2,8 d+4}$ is a subgraph of $K_{2}+(8 d+4) K_{1}$, but $H(17)$ is not a subgraph of $K_{2}+(8 d+4) K_{1}$.

Example $G_{1, \boldsymbol{d}}^{-}$: This is defined to be $P_{d+2}$ (the path with $d+2$ vertices), which is not $(1, d)^{-}$-colourable, and so not $(1, d)^{-}$-choosable. It cannot have as a minor any graph containing either a circuit or a vertex of degree $\geqslant 3$, which includes $H(i)$ for $i \in\{3,5, \ldots, 9,11, \ldots, 22\}$. Also, $G_{1,1}^{-}\left(P_{3}\right)$ does not have $H(4)\left(P_{4}\right)$ as a minor, and $G_{1,2}^{-}$ $\left(P_{4}\right)$ does not have $H(10)\left(P_{5}\right)$ as a minor.

Example $G_{k, d}^{-}(k=2,3)$ : Chartrand, Geller and Hedetniemi [1] showed how to construct, for each $d$, a graph that is planar, and hence $K_{5}$-minor-free, but not $(3, d)^{-}$colourable; we can take this as $G_{3, d}^{-}$. And they [2] and Woodall [11] gave different constructions for a graph that is outerplanar, and hence without $K_{4}$ and $K_{2,3}$ minors, that is not $(2, d)^{-}$-colourable; we can take this as $G_{2, d}^{-}$.

Example $\boldsymbol{V}_{\mathbf{4}, \mathbf{0}}$ : We need a graph that is planar, and hence $K_{5}$-minor-free, but not 4-choosable. Voigt [9] gave the first example of such a graph. Other examples are due to Gutner [4] and Mirzakhani [5].

\section{Arguments}

We will use the following four theorems; the first is already known, and the other three are proved in section 4 . Here $K_{5}-e$ denotes the graph obtained from $K_{5}$ by deleting one edge. A monochromatic $H$-minor is a monochromatic subgraph that contracts to $H$. 
Theorem 3.1. ([12], Theorem 3.5.) Let $H$ be a connected graph with at least one edge, and let $G$ be a $\left(K_{1}+H\right)$-minor-free graph. Suppose that each vertex $v$ of $G$ is given a list $L(v)$ of at least 2 colours. Then $G$ has an L-colouring with no monochromatic $H$-minor.

Theorem 3.2. Every $\left(K_{1}+\left(K_{1} \cup K_{1,2}\right)\right)$-minor-free graph is $(2,1)$-choosable.

Theorem 3.3. Every $\left(K_{5}-e\right)$-minor-free graph is 4 -choosable and $(3,1)$-choosable.

Theorem 3.4. Every $K_{5}$-minor-free graph is $(4,2)^{*}$-choosable.

We now summarize the arguments needed to prove the results listed in Table 1 . Note that if $H(i)$ is a minor of $H(j)$, then every $H(i)$-minor-free graph is also $H(j)$-minorfree, and so an argument $X_{k, d}$ that applies to $H(j)$-minor-free graphs will also apply to $H(i)$-minor-free graphs as well.

Arguments $\boldsymbol{O}_{1, d}, \boldsymbol{O}_{1, d}^{*}$ and $\boldsymbol{O}_{1, d}^{-}$: We use these names when none of our other arguments prove the result but the result is obvious anyway. For example, $O_{1,3}^{*}$ applied to $H(11)$ says that every $K_{1,4}$-minor-free graph can be 1 -coloured in such a way that there is no monochromatic $K_{1,4}$ subgraph.

Argument $\boldsymbol{H}_{\boldsymbol{k}, \mathbf{0}}$ : The choosability analogue of Hadwiger's conjecture, that every $K_{k+1^{-}}$ minor-free graph is $k$-choosable, is easy to prove if $k \leqslant 2$, and it holds if $k=3$ since $K_{4}$-minor-free graphs are 2-degenerate [3]. (As we have already seen, it does not hold if $k=4$.)

Argument $\boldsymbol{A}_{\mathbf{2}, \mathbf{1}}$ : Since $H(i) \subseteq H(16)=K_{1}+\left(K_{1} \cup K_{1,2}\right)$ if $i \in\{4, \ldots, 8,10, \ldots, 16\}$, Theorem 3.2 implies that, for these values of $i$, every $H(i)$-minor-free graph is $(2,1)$ choosable.

Arguments $\boldsymbol{B}_{\mathbf{3 , 1}}$ and $\boldsymbol{B}_{\mathbf{4}, \mathbf{0}}$ : Theorem 3.3 implies that every $H(i)$-minor-free graph $(1 \leqslant i \leqslant 29)$ is both $(3,1)$-choosable and $(4,0)$-choosable.

Argument $\boldsymbol{C}_{\mathbf{2}, \mathbf{2}}^{*}$ : Theorem 3.1 implies that every $\left(K_{1}+K_{1,3}\right)$-minor-free graph is $(2,2)^{*}$ choosable (i.e., with no monochromatic $K_{1,3}$-minor), and $H(23) \subset H(24)=K_{1}+K_{1,3}$.

Argument $\boldsymbol{C}_{\mathbf{2}, \mathbf{2}}^{-}$: Theorem 3.1 implies that every $\left(K_{1}+P_{4}\right)$-minor-free graph is $(2,2)^{-}$choosable (i.e., with no monochromatic $P_{4}$-minor), and $H(i) \subseteq H(22)=K_{1}+P_{4}$ if $17 \leqslant i \leqslant 22$.

Argument $\boldsymbol{D}_{\mathbf{4 , 2}}^{*}$ : This is exactly Theorem 3.4. 


\section{Proofs}

We first prove Theorem 3.2. We start by proving a lemma. A theta graph is a graph that is the union of three internally disjoint paths connecting the same two vertices. A bad edge is an edge whose endvertices have the same colour.

Lemma 4.1.1. (a) Let $G_{1}$ be a theta graph, let $L_{1}$ be a 2-list-assignment to $G_{1}$, and let a specified vertex $u$ of degree 2 in $G_{1}$ be precoloured with a colour from its list. Then this colouring of $u$ can be extended to an $\left(L_{1}, 1\right)$-colouring of $G_{1}$ in which $u$ is properly coloured (that is, $u$ has no neighbour with the same colour as itself).

(b) Every subdivision of $K_{4}$ is $(2,1)$-choosable.

Proof. (a) Let $G_{1}$ consist of three paths $P_{1}, P_{2}, P_{3}$ connecting two vertices $a$, $b$, and suppose that $u$ is in $P_{1}$. The remaining vertices of $P_{1}$ (including $a$ and $b$ ) can easily be coloured with no bad edges. Colour the internal vertices of $P_{2}$ and $P_{3}$ in order from $a$ towards $b$, and from $b$ towards $a$, respectively, so that there are at most two bad edges, namely the edge of $P_{2}$ incident with $b$ and the edge of $P_{3}$ incident with $a$. If the resulting colouring is not an $\left(L_{1}, 1\right)$-colouring then these edges are both bad and are adjacent to each other. Thus one of $P_{2}$ and $P_{3}$, say $P_{2}$, has length 1 , and the other, $P_{3}$, has length at least three (since for the edge of $P_{2}$ to be bad, $a$ and $b$ must have the same colour); so changing the colour of the vertex in $P_{3}$ adjacent to $a$ will create the required $\left(L_{1}, 1\right)$ colouring. (Clearly $u$ is properly coloured.)

(b) Let $G_{2}$ be a subdivision of $K_{4}$ and let $L_{2}$ be a 2-list-assignment to $G_{2}$. Let the vertices of degree 3 in $G_{2}$ be $a, b, c, d$, and let $C$ be the circuit containing $a, b$ and $c$ but not $d$. The vertices of $C$ can easily be coloured so that there is at most one bad edge. Now all remaining vertices other than $d$ can be coloured without introducing any more bad edges, and finally $d$ can be coloured so as to introduce at most one more bad edge. There are now at most two bad edges. If there are two, and they induce a monochromatic $K_{1,2}$, then change the colour of the middle vertex; since its degree is at most 3 , we now have at most one bad edge, and we have the required $\left(L_{2}, 1\right)$-colouring of $G_{2}$.

The following theorem implies Theorem 3.2, since $H(16)=K_{1}+\left(K_{1} \cup K_{1,2}\right)$.

Theorem 4.1. Let $G$ be an $H(16)$-minor-free graph, and let $L$ be a 2-list-assignment to $G$. Then $G$ is $(L, 1)$-colourable. Moreover, if $G$ is not a subdivision of $K_{4}$, and $u$ is a vertex that has degree at most 2 in each block that contains it, and $u$ is precoloured with a colour from its list, then this colouring of $u$ can be extended to an $(L, 1)$-colouring of $G$ in which $u$ is properly coloured.

Proof. There is no loss of generality in assuming that $G$ is connected. Suppose first that $G$ is a block (i.e., $G$ has no cutvertex). Then it is easy to see that $G$ has maximum degree at most three and is $K_{2}$, a circuit, a theta graph, or a subdivision of $K_{4}$. Thus the result follows from Lemma 4.1.1.

So suppose that $G$ has a cutvertex $x$, and note that $x$ has degree at most 2 in each block that contains it, since otherwise $G$ has an $H(16)$ minor. For the same reason, no 
block of $G$ is a subdivision of $K_{4}$. Let $G=G_{1} \cup G_{2}$, where $G_{1} \cap G_{2}=\{x\}, u \in G_{1}$ (possibly $u=x$ ), and $G_{i}$ is connected and has more than one vertex $(i=1,2)$. Then we may assume inductively that we can extend the given colouring of $u$ to an $(L, 1)$-colouring of $G_{1}$ in which $u$ is properly coloured (where by a slight abuse of terminology we write $L$ for the restriction of $L$ to $V\left(G_{1}\right)$ ), and we can extend the resulting colouring of $x$ to an $(L, 1)$-colouring of $G_{2}$ in which $x$ is properly coloured. The union of these two colourings is the required $(L, 1)$-colouring of $G$ in which $u$ is properly coloured.

We now prove the main structural lemma needed for the proof of Theorem 3.3.

Lemma 4.2.1. Let $G$ be a 3-connected $\left(K_{5}-e\right)$-minor-free graph. Then $G$ is either a wheel, or the triangular prism, or $K_{3,3}$.

Proof. We first need some notation and preliminary results. Suppose that $H$ is a wheel, or the triangular prism, or $K_{3,3}$, and that $G$ has a subgraph $H^{\prime}$ that is a subdivision of $H$, but $G$ is not isomorphic to $H$. If $a b$ is an edge of $H$, or $a b c$ is a triangle of $H$, then we denote by $P_{a b}$ or $T_{a b c}$ the subgraph of $H^{\prime}$ corresponding to the edge $a b$ or the triangle $a b c$, and refer to it as a subdivided edge or a subdivided triangle, respectively, of $H^{\prime}$ (even if the edge $a b$ or triangle $a b c$ has not in fact been subdivided). We will need the following results.

Claim 1. If $P_{a b}$ is a subdivided edge of length at least two in $H^{\prime}$ (i.e., the edge ab has really been subdivided), then there is a path $P$ in $G$ such that one endvertex of $P$ is an internal vertex of $P_{a b}$, the other endvertex is a vertex of $H^{\prime}$ that is not in $P_{a b}$, and no other vertex of $P$ is in $H^{\prime}$.

Proof. If there were no such path $P$, then $\{a, b\}$ would be a cutset of two vertices in $G$, which is impossible since $G$ is 3 -connected.

Claim 2. G has a minor that is isomorphic to a graph that can be obtained from $H$ in one of the following two ways:

(a) by adding a new edge $e_{1}$ that joins a vertex of a triangle in $H$ to a new vertex $v_{1}$ subdividing the opposite edge of the triangle;

(b) by adding a new edge $e_{1}$ that joins two nonadjacent vertices of $H$.

Proof. We consider three cases.

Case 1: $H^{\prime} \neq H$. Then there is a subdivided edge $P_{a b}$ of length at least two in $H^{\prime}$, and hence a path $P$ as in Claim 1. Then $G$ has a minor formed as in (a) if $P$ joins two vertices that are both in the same subdivided triangle of $H^{\prime}$, and formed as in (b) otherwise.

Case 2: $H^{\prime} \cong H$ and $|V(G)|=\left|V\left(H^{\prime}\right)\right|$. Since $G \varsubsetneqq H$ by the first paragraph in the proof of Lemma 4.2.1, there is an edge of $G$ joining two nonadjacent vertices of $H^{\prime}$, and so $G$ has a minor formed as in (b).

Case 3: $H^{\prime} \cong H$ and there is a vertex $v \in V(G) \backslash V\left(H^{\prime}\right)$. Since $G$ is 3-connected, there are three internally disjoint paths from $v$ to three vertices $a, b, c \in V\left(H^{\prime}\right)$. It is not possible 


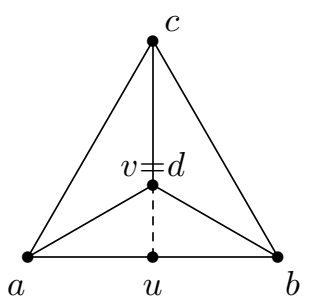

(a)

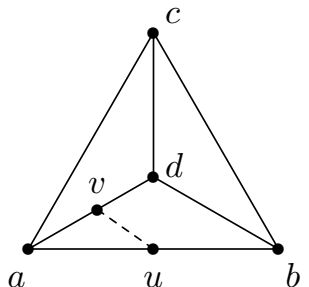

(b)

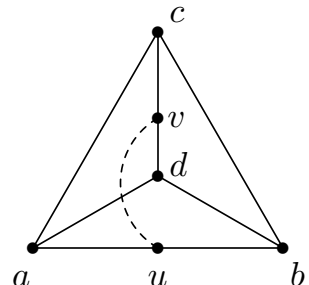

(c)

Fig. 1. Possible ways of adding a path to a subdivision of $K_{4}$.

that $a, b, c$ form a triangle in $H^{\prime}$, since this would imply that $H$ is a wheel or the triangular prism and hence that $G$ has $K_{5}-e$ as a minor. Thus some two of $a, b, c$ are nonadjacent and $G$ has a minor formed as in (b). This proves Claim 2.

We can now proceed with the proof of Lemma 4.2.1. Since $G$ is 3-connected, it has minimum degree at least 3 , and so contains a subgraph $H^{\prime}$ that is a subdivision of $K_{4}$ [3]. If $G \cong K_{4}$, which is the wheel $W_{3}$, then we are finished; so suppose $G ¥ K_{4}$. If $H^{\prime} \cong K_{4}$ then there is a vertex $v \in V(G) \backslash V\left(H^{\prime}\right)$ and, since $G$ is 3 -connected, $G$ contains three internally disjoint paths that connect $v$ to three vertices of $H^{\prime}$; but then $G$ has a $K_{5}-e$ minor, contrary to hypothesis. Thus we may assume that $H^{\prime} \neq K_{4}$.

Let the vertices of degree 3 in $H^{\prime}$ be $a, b, c, d$, and assume that $P_{a b}$ has length at least 2 . Let $P$ be a path, as in Claim 1, joining an internal vertex $u$ of $P_{a b}$ to a vertex $v$ of $H^{\prime}$ outside $P_{a b}$. If $v \in\{c, d\}$ then $H^{\prime} \cup P$ is a subdivision of $W_{4}$ (Fig. 1(a)). If $v$ is an internal vertex of any of the subdivided edges $P_{a c}, P_{a d}, P_{b c}$ and $P_{b d}$ then $H^{\prime} \cup P$ is a subdivision of the triangular prism (Fig. 1(b)). And if $v$ is an internal vertex of $P_{c d}$ then $H^{\prime} \cup P$ is a subdivision of $K_{3,3}$ (Fig. 1(c)). Assuming that the result of the lemma is false, we will obtain a contradiction by considering three cases.

Case 1: $G$ contains a subdivision of $W_{4}$. Choose $n$ maximal such that $G$ has a subgraph $W_{n}^{\prime}$ that is a subdivision of $W_{n}$. Let the vertices of degree 3 in $W_{n}^{\prime}$ be $a_{1}, \ldots, a_{n}$, and let the vertex of degree $n$ be $b$. If $G \cong W_{n}$ then we are finished; so suppose this is not the case. We want to obtain a contradiction.

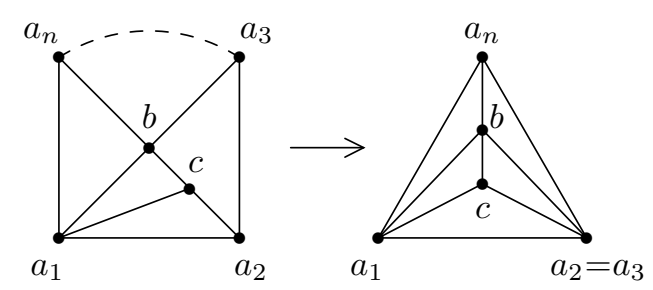

(a)

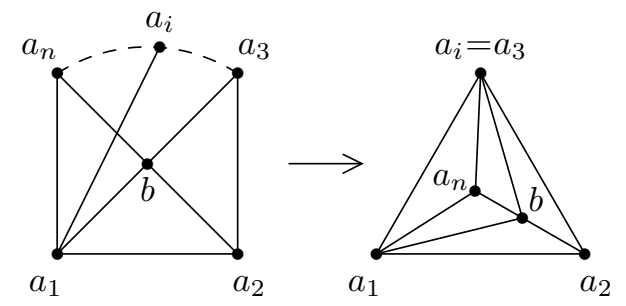

(b)

Fig. 2. Adding an edge to $W_{n}$ gives a $K_{5}-e$ minor. 
Suppose first that $G$ contains a path $P$, as in Claim 1, that joins two vertices in a subdivided triangle, say $T_{a_{1} a_{2} b}$, of $W_{n}^{\prime}$. If $P$ joins $b$ to a point on $P_{a_{1} a_{2}}$ then $G$ contains a subdivision of $W_{n+1}$, which contradicts the maximality of $n$. If not, then by contracting edges we form a subgraph isomorphic to $W_{n}+e_{1}$, where $e_{1}$ joins $a_{1}$ to a new vertex $c$ subdividing the edge $a_{2} b$ (Fig $2(\mathrm{a})$ ); then by contracting all edges of the path $a_{2} a_{3} \ldots a_{n-1}$ we obtain $K_{5}-e$ as a minor, which is again a contradiction.

So suppose that $G$ does not contain such a path $P$. Then, applying Claim 2 with $H=W_{n}, G$ has no minor formed as in Claim 2(a), and so it must have a minor formed as in Claim 2(b), by adding an edge joining two nonadjacent vertices of $W_{n}$; let these be $a_{1}$ and $a_{i}$, where $3 \leqslant i \leqslant n-1$ (Fig $2(\mathrm{~b})$ ). Then by contracting all edges of the paths $a_{3}, \ldots, a_{i}$ and $a_{i+1}, \ldots, a_{n}$ we again obtain $K_{5}-e$ as a minor. This contradiction completes the discussion of Case 1.

Case 2: $G$ has a subgraph $H^{\prime}$ that is a subdivision of the triangular prism $H$. Then $G$ has a minor isomorphic to a graph that is formed from $H$ as in Claim 2(a) or (b). In view of the symmetry of $H$, there are only two nonisomorphic graphs of this form, and Fig. 3 shows that both of them have $K_{5}-e$ as a minor, which is a contradiction.

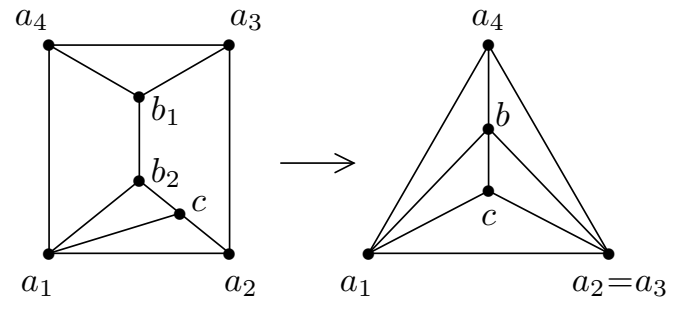

(a)

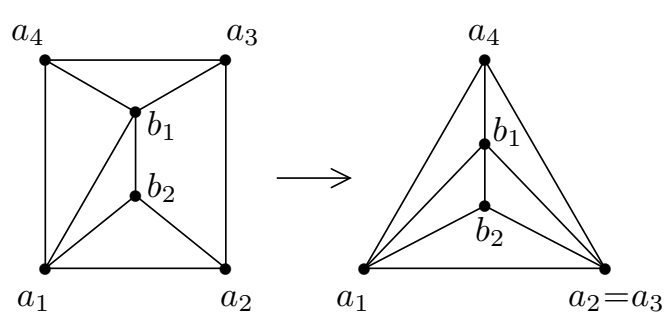

(b)

Fig. 3. Adding an edge to the triangular prism gives a $K_{5}-e$ minor.

Case 3: $G$ contains a subdivision of $K_{3,3}$. By Claim 2, $G$ has a minor isomorphic to a graph of the form $K_{3,3}+e_{1}$, where $e_{1}$ joins two nonadjacent vertices of $K_{3,3}$. If $e_{2}$ is any edge of $K_{3,3}$ not incident with either of these vertices, then contracting $e_{2}$ in $K_{3,3}+e_{1}$ gives $K_{5}-e$, again contradicting the fact that $G$ is $\left(K_{5}-e\right)$-minor-free. In every case we have a contradiction, and so the proof of Lemma 4.2.1 is complete.

If $U$ is a set of vertices of a graph $G$, we say that a colouring of $G$ is $U$-proper if no vertex in $U$ has any neighbour outside $U$ with the same colour as itself; this does not rule out the possibility that two adjacent vertices of $U$ (or two adjacent vertices outside $U$ ) may have the same colour as each other. If $u$ and $v$ are two adjacent vertices of $G$ that are precoloured, and every other vertex $x$ of $G$ is assigned a list $L(x)$ of colours, then by an $(L, d,\{u, v\})$-proper colouring of $G$ we mean a $\{u, v\}$-proper colouring in which each vertex $x \notin\{u, v\}$ receives a colour from its own list and has at most $d$ neighbours with the same colour as itself. (Even if $d=0$, this still allows $u$ and $v$ to have the same colour.)

The following theorem implies Theorem 3.3. 

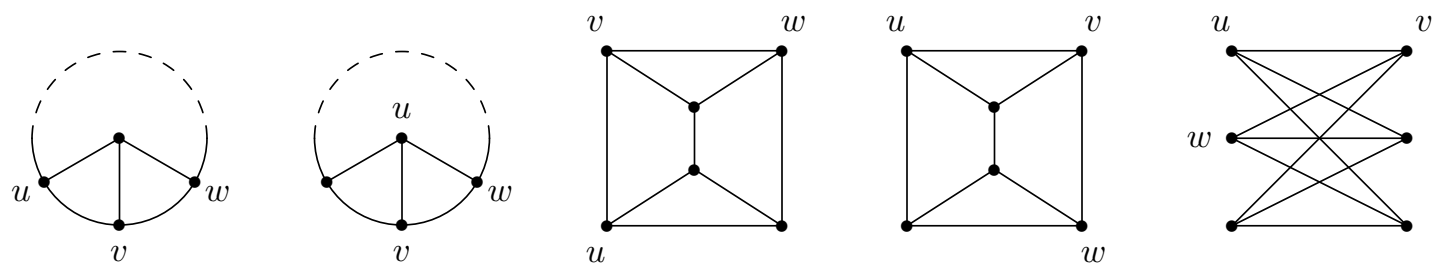

Fig. 4. Possible 3-connected $\left(K_{5}-e\right)$-minor-free graphs with two adjacent vertices $u, v$ precoloured.

Theorem 4.2. Let $(k, d) \in\{(4,0),(3,1)\}$, and let $G$ be a $\left(K_{5}-e\right)$-minor-free graph in which two adjacent vertices $u$ and $v$ are precoloured, and every other vertex $x$ is assigned a list $L(x)$ of $k$ colours. Then $G$ has an $(L, d,\{u, v\})$-proper colouring. (In other words, the remaining vertices can be coloured from their lists in such a way that each has at most $d$ neighbours with the same colour as itself, and the resulting colouring of $G$ is $\{u, v\}$-proper.)

Proof. There is no loss of generality in assuming that $G$ is connected. There are three cases to consider.

Case 1: $G$ is 3-connected. By Lemma 4.2.1, $G$ is either a wheel or the triangular prism or $K_{3,3}$. Taking account of the different possibilities for the vertices $u$ and $v$, we have one of the five cases in Fig. 4. In each case, with the vertex $w$ chosen as illustrated, $G-w$ is 2-degenerate, and indeed we can properly colour the uncoloured vertices of $G-w$ from their lists by ensuring that each has at most two coloured neighbours at the time it is coloured. If $k=4$ we can now colour $w$ properly. If $k=3$ then we can colour $w$ differently from any two of its neighbours, leaving at most one bad edge incident with $w$, which we can choose not to be the edge $v w$ or (if it exists) $u w$. This gives the required $(L, d,\{u, v\})$-proper colouring of $G$.

Case 2: $G$ is 2-connected but not 3-connected. Let $X=\{x, y\}$ be a cutset of two vertices and let $G_{1}$ and $G_{2}$ be subgraphs of $G$, each with at least three vertices, such that $G=G_{1} \cup G_{2}$ and $G_{1} \cap G_{2}$ is the subgraph induced by $X$. Since $u, v$ are adjacent, we may assume that they both lie in $G_{1}$.

If $x, y$ are nonadjacent let $G_{i}^{\prime}:=G_{i}+x y$, otherwise let $G_{i}^{\prime}:=G_{i}(i=1,2)$. Since $G$ is 2-connected, there is a path in $G_{2}$ from $x$ to $y$, as otherwise $x$ or $y$ would be a cutvertex of $G$. Thus $G_{1}^{\prime}$ is a minor of $G$, and so it is $\left(K_{5}-e\right)$-minor-free, and similarly so is $G_{2}^{\prime}$. We may assume inductively that the given colouring of $\{u, v\}$ can be extended to an $(L, d,\{u, v\})$-proper colouring of $G_{1}^{\prime}$, and that the resulting colouring of $\{x, y\}$ can be extended to an $(L, d,\{x, y\})$-proper colouring of $G_{2}^{\prime}$. The union of these two colourings is the required $(L, d,\{u, v\})$-proper colouring of $G$.

Case 3: $G$ is connected but not 2-connected. Let $x$ be a cutvertex of $G$ and let $G_{1}$ and $G_{2}$ be subgraphs of $G$, each with at least two vertices, such that $G=G_{1} \cup G_{2}$ and $G_{1} \cap G_{2}=\{x\}$. Since $u, v$ are adjacent, we may assume that they both lie in $G_{1}$. First inductively extend the given colouring of $\{u, v\}$ to an $(L, d,\{u, v\})$-proper colouring of $G_{1}$. Then colour an arbitrary neighbour $y$ of $x$ in $G_{2}$ with a colour from its list different from 
the colour of $x$. Finally, extend the resulting colouring of $\{x, y\}$ to an $(L, d,\{x, y\})$-proper colouring of $G_{2}$. The union of these two colourings is the required $(L, d,\{u, v\})$-proper colouring of $G$. This completes the proof of Theorem 4.2

Our final theorem implies Theorem 3.4.

Theorem 4.3. Let $G$ be a $K_{5}$-minor-free graph, let $L$ be a 4-list-assignment to $G$, let $U$ be a set of at most three mutually adjacent vertices in $G$, and suppose that the vertices of $U$ are all precoloured from their lists. Then this colouring of $U$ can be extended to a $U$-proper $(L, 2)^{*}$-colouring of $G$.

Proof. There is no loss of generality in assuming that $G$ is edge-maximal, that is, that the addition of any further edge to $G$ would create a $K_{5}$ minor. Wagner [10] proved that such a graph either is a maximal planar graph, or is a certain 3-regular triangle-free graph $V_{8}$, or has a cutset of at most three mutually adjacent vertices. ( $V_{8}$, a Möbius ladder, is obtained from a circuit of length eight by joining each pair of diagonally opposite vertices by a new edge.)

Suppose first that $G$ is a maximal planar graph (a triangulation). There is no loss of generality in assuming that $|U|=3$, say $U=\{u, v, w\}$. Let $L^{\prime}$ be obtained from $L$ by removing the given colour of $w$ from every other vertex; then $L^{\prime}$ is a 3-list-assignment to $G-w$. By ([12], Lemma 4.2), which says that a planar graph is $(3,2)^{*}$-choosable even if two adjacent vertices are precoloured, the given colouring of $u$ and $v$ can be extended to a $(u, v)$-proper $\left(L^{\prime}, 2\right)^{*}$-colouring of $G-w$, and this gives the required $U$-proper $(L, 2)^{*}$ colouring of $G$.

Suppose next that $G \cong V_{8}$. Since $G$ has maximum degree three, each uncoloured vertex can be coloured in turn to give a $U$-proper $(L, 2)^{*}$-colouring of $G$. (Indeed, this can be chosen to be an $(L, 1)$-colouring, or even an $(L, 0)$-colouring unless $|U|=2$ and the two vertices of $U$ have the same colour.)

Suppose finally that $G$ has a cutset $X$ consisting of at most three mutually adjacent vertices. Let $G_{1}$ and $G_{2}$ be subgraphs of $G$, each with at least $|X|+1$ vertices, such that $G=G_{1} \cup G_{2}$ and $G_{1} \cap G_{2}$ is the subgraph induced by $X$. Since all vertices in $U$ are mutually adjacent, we may assume that $U \subseteq V\left(G_{1}\right)$. We may assume inductively that the given colouring of $U$ can be extended to a $U$-proper $(L, 2)^{*}$-colouring of $G_{1}$, and that the resulting colouring of $X$ can be extended to an $X$-proper $(L, 2)^{*}$-colouring of $G_{2}$, and the union of these two colourings is the required $U$-proper $(L, 2)^{*}$-colouring of $G$.

\section{References}

[1] G. Chartrand, D. P. Geller and S. Hedetniemi, A generalization of the chromatic number, Proc. Cambridge Philos. Soc. 64 (1968) 265-271.

[2] G. Chartrand, D. Geller and S. Hedetniemi, Graphs with forbidden subgraphs. J. Combin. Theory Ser. B 10 (1971) 12-41.

[3] G. A. Dirac, A property of 4-chromatic graphs and some remarks on critical graphs, J. London Math. Soc. 27 (1952) 85-92. 
[4] S. Gutner, The complexity of planar graph choosability, Discrete Math. 159 (1996) 119-130.

[5] M. Mirzakhani, A small non-4-choosable planar graph, Bull Inst. Combin. Appl. 17 (1996) 15-18.

[6] R. Škrekovski, Choosability of $K_{5}$-minor-free graphs, Discrete Math. 190 (1998) 223226.

[7] R. Škrekovski, List improper colourings of planar graphs, Combin. Probab. Comput. 8 (1999) 293-299.

[8] C. Thomassen, Every planar graph is 5-choosable, J. Combin. Theory Ser. B 62 (1994) 180-181.

[9] M. Voigt, List colourings of planar graphs, Discrete Math. 120 (1993) 215-219.

[10] K. Wagner, Über eine Eigenschaft der ebenen Komplexe, Math. Ann. 114 (1937) 570-590.

[11] D. R. Woodall, Improper colourings of graphs, In: R. Nelson and R. J. Wilson (Eds.), Graph Colourings, Pitman Research Notes in Math, 218, Longman, Harlow, Essex, 1990, 45-63.

[12] D. R. Woodall, List Colourings of Graphs, in: J. W. P. Hirschfeld (Ed), Surveys in Combinatorics, 2001, London Math. Soc. Lecture Note Series 288, Cambridge University Press, 2001, 269-301. 